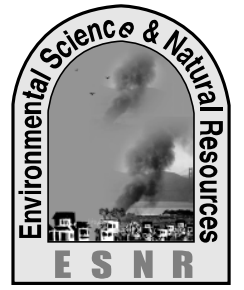

J. Environ. Sci. \& Natural Resources, 6(1): 59 - 67, 2013

ISSN 1999-7361

\title{
Physico-chemical properties of Dhepa River in Dinajpur District of Bangladesh
}

\author{
K. Rakiba and Z. Ferdoushi
}

Department of Fisheries Management, Hajee Mohammad Danesh Science and Technology University

\begin{abstract}
Among different water quality parameters dissolved oxygen, transparency, $\mathrm{pH}, \mathrm{PO}_{4}-\mathrm{P}$ and depth varied significantly among the sampling sites. The $\mathrm{pH}$ value in the present investigation remained a buffer condition (6.50- 7.90). Dissolved oxygen was ranges from 3.80 to $11.60 \mathrm{mg} / \mathrm{l}$ throughout the study periods. $\mathrm{PO}_{4}-\mathrm{P}$ concentration was observed highest $(0.30 \mathrm{mg} / \mathrm{l})$ in sampling site 2. On the basis of physical, chemical aspects sampling site 3 and sampling site 5 (situated in gosaipur and chandandoho) found in better condition in terms of limnological aspects. However, it could be concluded that Dhepa River will play important role in riverine fisheries and for further fisheries management.
\end{abstract}

Key words: Chemical properties, River water, Water quality

\section{Introduction}

Water is one of the most important compounds to the ecosystem. Better quality of water is described by its suitable physical, chemical and biological characteristics. But some correlation was possible among these parameters and the significant one would be useful to indicate quality of water (Manjare et.al., 2010). In freshwater ecosystems, it is well recognized that the biotic and physical attributes of habitats have a major influence on the diversity, distribution, and survival of organisms. Changes in nature of freshwater habitats can cause rapid changes in biodiversity composition, (De Pauw and Vanhooren, 1983). Plankton abundance depends on the physicochemical characteristics of the water body. The maximum productivity may be obtained when the physical and chemical parameters are at the optimum level. Therefore, water quality is a paramount factor in ecosystem productivity (Huet, 1986).

Main rivers of Dinajpur districts are: Dhepa, Punarbhaba and Atrai. The Dhepa River is enriched with many ecosystem services and has substantial impact on the ecology, biodiversity and socioeconomy of the surrounding localities. This resource is a critical source of protein for the people who live around the river. It plays an important role in the regional economy and food security of the local people. But at present the status of Dhepa river ecosystem is not very encouraging. Some parts of the river are completely dried up during winter season putting the ecosystem under threat. Moreover, unplanned urban and agricultural developments and the related anthropogenic disturbances, predominantly throwing of garbage, discharge of sewage and municipal wastes into waterbody, unload of sand, overexploitation of aquatic resources are reported as increasing problems responsible for destroying the overall ecosystem of Dhepa River. Therefore due to the importance of the riverine fishery, it is urgent need to highlight the linkages among river ecology, biodiversity, livelihoods and sustainable development in order to protect the river for future generations.

Increased human population, industrialization, use of fertilizers in agricultural land and various man-made activities are causing heavy and varied pollution in aquatic environment leading to water quality and depletion of aquatic biota. Unfortunately no data on the limnological aspects of Dhepa River is available because this river has never been extensively studied. Therefore, considering the significance of Dhepa River particularly in the field of ecology, the present study was undertaken to provide baseline data and determine their occurrence for management decision in the management of the fishery.

\section{Materials and Methods}

\section{Site selection}

River Dhepa in Dinajpur district was selected for the purpose of the study. It is located in Dinajpur district within latitudes $25^{\circ} 38^{\prime} \mathrm{N}$ and $25^{\circ} 53^{\prime} \mathrm{N}$ and longitudes $88^{\circ} 37^{\prime} \mathrm{E}$ and $88^{\circ} 43^{\prime} \mathrm{E}$. The river originated from the right-bank of the Atrai near Mohanpur and flowing southeast direction. Then it turned south near Setabganj, Bochaganj and then it falls into the Punarbhaba river.

\section{Experimental design}

Six sampling sites were established along the length of Dhepa River. Sites were chosen in such a manner to provide for even distance for effective sampling. The distance from each site was $1 \mathrm{~km}$. Figure 1 is showing the location of the experimental river with the sampling site. The sampling procedure was carried out in Dhepa River from September 2011 to April 2012. 


\section{Sample collection}

The water samples from Dhepa River were collected from six sites between 8 to 10 hours, in plastic bottles fortnightly. The water samples were immediately brought in to Laboratory for the estimation of various physico-chemical parameters like total alkalinity, nitrate-nitrogen and phosphate-phosphorus. Water temperature, air temperature, depth, $\mathrm{pH}$, dissolved oxygen and transparency were recorded at the time of sample collection, by using Thermometer, Digital Dissolved Oxygen Meter and $\mathrm{pH}$ Meter. Transparency was measured with the help of Secchi Disc. While other parameters such as Alkalinity, Phosphate and Nitrate were estimated by using Standard Methods as prescribed by APHA (1992) and HACH Kit.

\section{Statistical Analysis}

The comparison between means \pm SD (standard deviation) was tested for significance by using oneway ANOVA analysis and Tukey's test. The statistical analyses were calculated, using SPSS.

\section{Results}

Water quality parameters of a large number of samples were analyzed in this experiment to observe any appreciable changes that might have occurred in response to different sampling sites. Various physical and chemical parameters of Dhepa River were determined under six sampling sites (St.1, St.2, St.3, St.4, St.5, and St.6). Physical parameters such as air temperature, water temperature, transparency, depth and chemical parameters such as dissolved oxygen, $\mathrm{pH}$, nitrate, phosphate and alkalinity were measured fortnightly. The average values of water quality parameters of different sites are presented in Table 1 and Table 2.

\section{Physical Parameters}

\section{Air Temperature}

The range of air temperature was found to vary from 18.60 to $34.10{ }^{\circ} \mathrm{C}, 18.00$ to $35.30{ }^{\circ} \mathrm{C}, 22.00$ to 36.20 ${ }^{\circ} \mathrm{C}, 23.10$ to $36.00{ }^{\circ} \mathrm{C}, 22.70$ to $36.00{ }^{\circ} \mathrm{C}$ and 23.00 to $35.93{ }^{\circ} \mathrm{C}$ during the experiment in sampling sites St.1, St.2, St.3, St.4, St.5, and St.6, respectively (Table 3). The mean $( \pm \mathrm{SD})$ values of air temperature were 26.93 $\pm 4.87{ }^{\circ} \mathrm{C}, 27.98 \pm 5.17{ }^{\circ} \mathrm{C}, 29.62 \pm 4.27{ }^{\circ} \mathrm{C}, 29.20 \pm$ $4.13{ }^{\circ} \mathrm{C}, 29.49 \pm 4.35{ }^{\circ} \mathrm{C}$ and $30.33 \pm 4.16{ }^{\circ} \mathrm{C}$ in sites St.1, St.2, St.3, St.4, St.5, and St.6, respectively (Table 3). The maximum air temperature $\left(36.20{ }^{\circ} \mathrm{C}\right)$ was found in March in St.3, while minimum $\left(18{ }^{\circ} \mathrm{C}\right)$ air temperature was found in December in St.2. There was significant difference $(P<0.05)$ in St. 1 with St.3 and St.6 but there was no significant different among St.2, St.4 and St.5 (Table 4).

\section{Water Temperature}

Water temperature plays a vital role in aquatic production through influencing physical, chemical and biological conditions of a water body. The water temperature was found to vary from 17.30 to 33.20 ${ }^{\circ} \mathrm{C}, 17.00$ to $32.50{ }^{\circ} \mathrm{C}, 20.30$ to $33.10{ }^{\circ} \mathrm{C}, 18.40$ to $33.30{ }^{\circ} \mathrm{C}, 18.30$ to $33.50{ }^{\circ} \mathrm{C}$ and 18.50 to $33.20{ }^{\circ} \mathrm{C}$ during the experiment in sampling sites St.1, St.2, St.3, St.4, St.5 and St.6, respectively (Table 3). The mean $( \pm \mathrm{SD})$ values of water temperature were 25.60 $\pm 4.94{ }^{\circ} \mathrm{C}, 25.50 \pm 4.98{ }^{\circ} \mathrm{C}, 26.66 \pm 4.27{ }^{\circ} \mathrm{C}, 26.58 \pm$ $4.54{ }^{\circ} \mathrm{C}, 26.53 \pm 4.84{ }^{\circ} \mathrm{C}$ and $26.54 \pm 4.61{ }^{\circ} \mathrm{C}$ in sites St.1, St.2, St.3, St.4, St.5, and St.6, respectively (Table 3). The maximum water temperature $(33.50$ ${ }^{\circ} \mathrm{C}$ ) was found in April in St.5, while minimum (17.00 ${ }^{\circ} \mathrm{C}$ ) water temperature was found in December in St.2. There was no significant difference $(P>0.05)$ among different sites, when statistical analysis (ANOVA) was performed (Table 4).

\section{Transparency}

Highest $(48.70 \mathrm{~cm})$ and lowest $(8.10 \mathrm{~cm})$ transparency was found in St.2 in February and September respectively (Figure 4). Transparency was found to vary from 8.90 to $39.80 \mathrm{~cm}, 8.10$ to 48.70 $\mathrm{cm}, 9.10$ to $44.60 \mathrm{~cm}, 9.10$ to $38.00 \mathrm{~cm}, 9.40$ to 36.90 $\mathrm{cm}$ and 8.20 to $31.50 \mathrm{~cm}$ during the experiment in sampling sites St.1, St.2, St.3, St.4, St.5, and St.6, respectively (Table 3$)$. The mean $( \pm \mathrm{SD})$ values of transparency were $22.17 \pm 8.70 \mathrm{~cm}, 29.34 \pm 11.97$ $\mathrm{cm}, 22.18 \pm 12.98 \mathrm{~cm}, 21.39 \pm 9.34 \mathrm{~cm}, 23.94 \pm 7.39$ $\mathrm{cm}$ and $18.94 \pm 5.82 \mathrm{~cm}$ in sites St.1, St.2, St.3, St.4, St.5, and St.6, respectively (Table 3). Significant different was shown in St.2 with St.1, St.3, St.4 and St.6. There was no significant difference shown between St.2 and St.5 (Table 4).

\section{Depth}

Significant difference $(P<0.05)$ in water depth was observed between St.1 and St.5 (Table 4). The water level was found to vary from 2.00 to $7.60 \mathrm{ft}, 2.50$ to $12.80 \mathrm{ft}, 3.10$ to $16.00 \mathrm{ft}, 2.00$ to $15.00 \mathrm{ft}, 3.00$ to $18.60 \mathrm{ft}$ and 2.00 to $11.20 \mathrm{ft}$ during the experiment in sampling sites St.1, St.2, St.3, St.4, St.5, and St.6, respectively (Table 3$)$. The mean $( \pm \mathrm{SD})$ values of water depth were $3.76 \pm 2.05 \mathrm{ft}, 6.81 \pm 3.09 \mathrm{ft}, 7.96 \pm$ $4.05 \mathrm{ft}, 7.58 \pm 4.17 \mathrm{ft}, 10.82 \pm 4.68 \mathrm{ft}$ and $5.14 \pm 2.77$ $\mathrm{ft}$ in sites St.1, St.2, St.3, St.4, St.5, and St.6, respectively (Table 3 ). 
Table 1 : Average values of different physical parameters of Dhepa river in different sampling sites during the period of study

\begin{tabular}{|c|c|c|c|c|c|c|c|c|c|c|c|c|c|c|c|c|c|}
\hline \multirow[t]{2}{*}{ Parameters } & \multirow[t]{2}{*}{ Site } & \multicolumn{16}{|c|}{ Average values of physical parameters } \\
\hline & & Sep & Sep & Oct & Oct & Nov & Nov & Dec & Dec & Jan & Jan & Feb & Mar & Mar & Mar & Apr & Apr \\
\hline \multirow{6}{*}{$\begin{array}{c}\text { Air temperature } \\
\left({ }^{\circ} \mathrm{C}\right)\end{array}$} & 1 & 34.10 & 32.27 & 30.13 & 27.00 & 25.00 & 28.90 & 26.70 & 18.60 & 18.70 & 26.00 & 20.90 & 24.80 & 33.60 & 27.70 & 32.17 & 24.30 \\
\hline & 2 & 34.00 & 32.67 & 31.33 & 29.23 & 25.10 & 30.00 & 24.30 & 18.00 & 21.10 & 24.53 & 22.00 & 24.90 & 33.50 & 29.90 & 31.80 & 35.30 \\
\hline & 3 & 35.20 & 32.50 & 34.53 & 30.00 & 24.80 & 31.33 & 27.00 & 22.00 & 25.80 & 29.00 & 24.80 & 26.00 & 36.20 & 28.70 & 32.40 & 33.70 \\
\hline & 4 & 36.00 & 32.50 & 30.77 & 32.50 & 29.60 & 32.50 & 25.70 & 23.10 & 25.30 & 28.70 & 24.10 & 24.30 & 26.60 & 28.00 & 33.10 & 34.50 \\
\hline & 5 & 36.00 & 35.97 & 30.47 & 31.00 & 29.80 & 31.80 & 26.50 & 22.70 & 24.00 & 29.30 & 23.60 & 24.90 & 28.70 & 29.10 & 32.80 & 35.30 \\
\hline & 6 & 35.00 & 35.93 & 32.47 & 32.33 & 31.40 & 28.93 & 26.50 & 23.00 & 25.50 & 29.20 & 25.30 & 25.60 & 32.00 & 33.30 & 33.90 & 35.00 \\
\hline \multirow{6}{*}{$\begin{array}{c}\text { Water } \\
\text { temperature }\left({ }^{\circ} \mathbf{C}\right)\end{array}$} & 1 & 31.30 & 30.20 & 29.50 & 26.60 & 22.90 & 24.20 & 19.10 & 17.30 & 18.00 & 22.10 & 22.40 & 24.00 & 30.20 & 29.10 & 29.50 & 33.20 \\
\hline & 2 & 31.00 & 30.30 & 29.30 & 26.30 & 23.00 & 23.50 & 18.60 & 17.00 & 18.60 & 22.90 & 21.40 & 23.80 & 30.60 & 30.40 & 29.30 & 32.50 \\
\hline & 3 & 32.00 & 30.60 & 29.70 & 28.60 & 24.20 & 26.60 & 21.90 & 20.30 & 20.70 & 22.70 & 21.30 & 25.10 & 31.10 & 29.00 & 29.60 & 33.10 \\
\hline & 4 & 32.30 & 30.60 & 28.10 & 28.70 & 23.40 & 24.60 & 19.90 & 18.40 & 20.60 & 26.80 & 21.90 & 26.70 & 28.60 & 31.20 & 30.10 & 33.30 \\
\hline & 5 & 33.00 & 31.90 & 28.90 & 28.10 & 22.30 & 24.80 & 19.30 & 18.30 & 20.20 & 26.20 & 22.00 & 26.20 & 28.90 & 30.40 & 30.50 & 33.50 \\
\hline & 6 & 33.20 & 31.40 & 30.00 & 28.30 & 23.50 & 24.70 & 19.20 & 18.50 & 20.80 & 27.00 & 22.00 & 24.80 & 29.30 & 29.70 & 30.20 & 32.10 \\
\hline \multirow{6}{*}{$\begin{array}{c}\text { Transparency } \\
\text { (cm) }\end{array}$} & 1 & 8.90 & 9.45 & 23.95 & 20.55 & 23.15 & 21.00 & 30.50 & 14.00 & 20.50 & 29.00 & 39.80 & 29.60 & 29.00 & 30.20 & 9.65 & 16.10 \\
\hline & 2 & 8.10 & 9.00 & 28.05 & 24.55 & 30.25 & 32.00 & 34.50 & 44.50 & 31.00 & 30.50 & 48.70 & 34.80 & 40.00 & 42.00 & 10.65 & 21.00 \\
\hline & 3 & 9.10 & 10.10 & 22.15 & 18.05 & 23.25 & 14.00 & 10.00 & 11.00 & 11.00 & 35.00 & 44.60 & 35.00 & 42.00 & 43.50 & 13.50 & 12.75 \\
\hline & 4 & 9.10 & 10.10 & 19.60 & 17.45 & 21.40 & 24.50 & 36.00 & 19.50 & 32.00 & 26.00 & 34.50 & 38.00 & 13.00 & 11.00 & 14.00 & 16.00 \\
\hline & 5 & 9.40 & 10.60 & 23.10 & 20.05 & 30.10 & 22.00 & 25.00 & 22.00 & 31.50 & 30.50 & 32.20 & 36.90 & 29.00 & 22.00 & 20.20 & 18.75 \\
\hline & 6 & 8.20 & 9.50 & 22.05 & 16.95 & 16.15 & 27.00 & 14.00 & 31.50 & 22.00 & 12.50 & 20.50 & 22.20 & 21.50 & 19.30 & 18.20 & 21.00 \\
\hline \multirow{6}{*}{$\begin{array}{l}\text { Depth } \\
\text { (ft) }\end{array}$} & 1 & 7.60 & 7.00 & 6.00 & 5.00 & 3.00 & 2.00 & 2.00 & 2.00 & 3.00 & 2.00 & 6.00 & 4.00 & 3.00 & 3.00 & 2.50 & 2.00 \\
\hline & 2 & 12.80 & 12.00 & 11.00 & 10.00 & 7.00 & 6.00 & 6.00 & 5.40 & 5.00 & 6.00 & 7.00 & 6.00 & 5.00 & 4.50 & 2.80 & 2.50 \\
\hline & 3 & 16.00 & 15.20 & 13.00 & 12.00 & 9.00 & 8.00 & 8.00 & 7.60 & 6.00 & 5.00 & 7.00 & 4.00 & 5.00 & 4.80 & 3.10 & 3.70 \\
\hline & 4 & 15.00 & 14.20 & 13.00 & 12.00 & 10.00 & 7.00 & 7.00 & 6.50 & 8.00 & 7.00 & 6.00 & 5.00 & 3.00 & 3.00 & 2.00 & 2.50 \\
\hline & 5 & 18.60 & 18.00 & 16.00 & 15.00 & 13.00 & 12.00 & 12.00 & 12.00 & 10.00 & 10.00 & 10.00 & 8.00 & 6.00 & 5.00 & 3.00 & 4.50 \\
\hline & 6 & 11.20 & 10.00 & 8.00 & 7.00 & 6.00 & 5.50 & 5.00 & 5.00 & 4.60 & 4.00 & 3.80 & 3.00 & 2.80 & 2.00 & 2.00 & 2.40 \\
\hline
\end{tabular}


Table 2 : Average values of different chemical parameters of Dhepa River in different sampling sites during the period of study

\begin{tabular}{|c|c|c|c|c|c|c|c|c|c|c|c|c|c|c|c|c|c|}
\hline \multirow[t]{2}{*}{ Parameters } & \multirow[t]{2}{*}{ Site } & \multicolumn{16}{|c|}{ Average values of chemical parameters } \\
\hline & & Sep & Sep & Oct & Oct & Nov & Nov & Dec & Dec & Jan & Jan & Feb & Mar & Mar & Mar & Apr & Apr \\
\hline \multirow{6}{*}{$\begin{array}{c}\text { Dissolved } \\
\text { oxygen (mg/l) }\end{array}$} & 1 & 5.60 & 5.80 & 6.50 & 6.80 & 8.30 & 8.50 & 8.60 & 9.50 & 8.00 & 7.70 & 6.70 & 4.20 & 6.20 & 7.90 & 6.40 & 8.50 \\
\hline & 2 & 5.50 & 6.40 & 9.00 & 5.90 & 5.90 & 6.60 & 8.10 & 8.50 & 7.40 & 6.30 & 8.00 & 3.80 & 5.50 & 7.40 & 6.80 & 8.30 \\
\hline & 3 & 6.10 & 5.70 & 7.30 & 7.20 & 7.90 & 8.70 & 10.60 & 9.80 & 10.20 & 7.30 & 8.70 & 4.50 & 4.90 & 7.30 & 7.40 & 9.20 \\
\hline & 4 & 6.20 & 5.70 & 6.60 & 6.80 & 9.00 & 8.60 & 9.00 & 11.60 & 8.30 & 7.20 & 6.00 & 7.80 & 5.60 & 8.10 & 7.30 & 7.80 \\
\hline & 5 & 6.50 & 6.70 & 6.80 & 6.70 & 7.10 & 7.30 & 9.50 & 8.80 & 8.50 & 7.40 & 5.70 & 8.10 & 7.00 & 7.50 & 6.90 & 8.00 \\
\hline & 6 & 6.40 & 6.20 & 6.70 & 6.70 & 8.90 & 7.20 & 10.40 & 10.00 & 11.40 & 9.90 & 7.40 & 8.10 & 7.20 & 7.60 & 7.40 & 8.40 \\
\hline \multirow{6}{*}{ pH } & 1 & 7.20 & 7.30 & 6.80 & 7.40 & 7.30 & 7.20 & 7.10 & 7.00 & 7.20 & 7.30 & 7.30 & 7.60 & 7.00 & 7.30 & 7.40 & 7.10 \\
\hline & 2 & 7.10 & 7.20 & 7.60 & 7.50 & 7.80 & 7.50 & 7.60 & 7.40 & 7.50 & 7.70 & 7.80 & 7.90 & 7.20 & 7.10 & 6.80 & 6.90 \\
\hline & 3 & 7.10 & 6.80 & 7.20 & 7.40 & 7.50 & 7.10 & 7.30 & 7.40 & 7.40 & 7.50 & 7.60 & 7.90 & 7.30 & 7.10 & 6.60 & 6.90 \\
\hline & 4 & 7.10 & 7.00 & 7.30 & 7.50 & 7.40 & 7.30 & 7.40 & 7.40 & 7.20 & 7.30 & 7.50 & 7.60 & 7.90 & 7.30 & 7.10 & 6.80 \\
\hline & 5 & 6.80 & 6.50 & 7.20 & 7.20 & 7.10 & 7.20 & 7.10 & 7.40 & 7.40 & 7.50 & 7.60 & 7.50 & 7.80 & 7.10 & 6.80 & 6.90 \\
\hline & 6 & 7.20 & 7.30 & 7.40 & 7.40 & 7.50 & 7.40 & 7.40 & 7.40 & 7.80 & 7.90 & 7.50 & 7.40 & 7.50 & 7.40 & 6.50 & 6.80 \\
\hline \multirow{6}{*}{$\begin{array}{c}\text { Alkalinity } \\
\text { (mg/l) }\end{array}$} & 1 & 38.00 & 44.00 & 40.00 & 46.00 & 50.00 & 42.00 & 46.00 & 46.00 & 44.00 & 46.00 & 54.00 & 57.00 & 44.60 & 40.00 & 21.00 & 34.00 \\
\hline & 2 & 36.00 & 34.00 & 46.00 & 44.00 & 50.00 & 44.00 & 46.00 & 42.00 & 44.00 & 48.00 & 65.00 & 48.00 & 42.00 & 32.00 & 17.00 & 32.00 \\
\hline & 3 & 30.00 & 26.00 & 42.00 & 44.00 & 52.00 & 40.00 & 48.00 & 44.00 & 44.00 & 50.00 & 57.00 & 54.00 & 42.60 & 32.00 & 19.00 & 34.00 \\
\hline & 4 & 32.00 & 30.00 & 38.00 & 46.00 & 44.00 & 40.00 & 42.00 & 44.00 & 44.00 & 42.00 & 46.00 & 48.00 & 61.00 & 38.00 & 23.00 & 32.00 \\
\hline & 5 & 28.00 & 26.00 & 42.00 & 42.00 & 40.00 & 42.00 & 40.00 & 44.00 & 44.00 & 48.00 & 52.00 & 50.00 & 57.00 & 34.00 & 21.00 & 32.00 \\
\hline & 6 & 32.00 & 34.00 & 40.00 & 38.00 & 48.00 & 40.00 & 42.00 & 44.00 & 48.00 & 52.00 & 54.00 & 52.00 & 57.00 & 38.00 & 15.00 & 28.00 \\
\hline \multirow{6}{*}{$\mathrm{NO}_{3}-\mathrm{N}(\mathrm{mg} / \mathrm{l})$} & 1 & 4.40 & 4.40 & 4.40 & 4.40 & 8.80 & 8.80 & 6.60 & 8.80 & 8.80 & 4.40 & 8.80 & 6.16 & 5.28 & 5.28 & 5.44 & 4.84 \\
\hline & 2 & 4.84 & 4.44 & 4.44 & 4.44 & 8.80 & 8.80 & 6.60 & 8.80 & 8.80 & 8.80 & 13.20 & 6.60 & 1.76 & 4.40 & 5.28 & 8.36 \\
\hline & 3 & 4.40 & 4.40 & 4.40 & 4.40 & 8.80 & 8.80 & 6.60 & 8.80 & 8.80 & 8.80 & 13.20 & 5.72 & 8.88 & 8.88 & 5.72 & 6.60 \\
\hline & 4 & 5.72 & 4.40 & 4.40 & 4.40 & 8.80 & 8.80 & 6.60 & 8.80 & 8.80 & 8.80 & 10.12 & 7.48 & 8.80 & 8.80 & 6.60 & 5.28 \\
\hline & 5 & 4.40 & 4.40 & 4.40 & 4.40 & 8.80 & 8.80 & 6.60 & 8.80 & 8.80 & 8.80 & 6.60 & 7.48 & 8.88 & 6.60 & 5.28 & 5.72 \\
\hline & 6 & 4.84 & 4.40 & 4.40 & 4.40 & 8.80 & 8.80 & 6.60 & 8.80 & 8.80 & 8.80 & 7.04 & 6.16 & 6.60 & 7.48 & 6.60 & 4.40 \\
\hline \multirow{6}{*}{$\mathrm{PO}_{4}-\mathrm{P}(\mathrm{mg} / \mathrm{l})$} & 1 & 0.14 & 0.15 & 0.18 & 0.08 & 0.30 & 0.14 & 0.10 & 0.08 & 0.12 & 0.10 & 0.04 & 0.18 & 0.12 & 0.12 & 0.04 & 0.16 \\
\hline & 2 & 0.04 & 0.03 & 0.20 & 0.08 & 0.30 & 0.09 & 0.14 & 0.09 & 0.12 & 0.17 & 0.04 & 0.14 & 0.19 & 0.10 & 0.04 & 0.09 \\
\hline & 3 & 0.03 & 0.05 & 0.19 & 0.09 & 0.20 & 0.29 & 0.24 & 0.08 & 0.08 & 0.17 & 0.18 & 0.27 & 0.12 & 0.06 & 0.08 & 0.15 \\
\hline & 4 & 0.08 & 0.04 & 0.20 & 0.08 & 0.21 & 0.08 & 0.12 & 0.07 & 0.12 & 0.14 & 0.03 & 0.06 & 0.14 & 0.08 & 0.02 & 0.19 \\
\hline & 5 & 0.10 & 0.04 & 0.17 & 0.10 & 0.21 & 0.08 & 0.14 & 0.07 & 0.12 & 0.14 & 0.01 & 0.14 & 0.08 & 0.14 & 0.03 & 0.15 \\
\hline & 6 & 0.04 & 0.03 & 0.20 & 0.08 & 0.18 & 0.14 & 0.16 & 0.09 & 0.14 & 0.16 & 0.02 & 0.16 & 0.03 & 0.18 & 0.03 & 0.21 \\
\hline
\end{tabular}


Table 3: Mean values $( \pm \mathrm{SD})$ and ranges of water quality parameters at different sampling sites throughout the period of study

\begin{tabular}{|c|c|c|c|c|c|c|}
\hline \multirow[b]{2}{*}{ Parameters } & \multicolumn{6}{|c|}{ Sampling sites } \\
\hline & Site 1 & Site 2 & Site 3 & Site 4 & Site 5 & Site 6 \\
\hline $\begin{array}{c}\text { Air temperature } \\
\text { (C) }\end{array}$ & $\begin{array}{c}26.93 \pm 4.87 \\
(18.60- \\
34.10)\end{array}$ & $\begin{array}{c}27.98 \pm 5.17 \\
(18.00- \\
35.30)\end{array}$ & $\begin{array}{c}29.62 \pm 4.27 \\
(22.00- \\
36.20)\end{array}$ & $\begin{array}{c}29.20 \pm 4.13 \\
(23.10- \\
36.00)\end{array}$ & $\begin{array}{c}29.49 \pm 4.35 \\
(22.70-36.00)\end{array}$ & $\begin{array}{c}30.33 \pm 4.16 \\
(23.00-35.93)\end{array}$ \\
\hline $\begin{array}{c}\text { Water } \\
\text { temperature }\left({ }^{\circ} \mathrm{C}\right)\end{array}$ & $\begin{array}{c}25.60 \pm 4.94 \\
(17.30- \\
33.20)\end{array}$ & $\begin{array}{c}25.53 \pm 4.98 \\
(17.00- \\
32.50)\end{array}$ & $\begin{array}{c}26.66 \pm 4.27 \\
(20.30- \\
33.10)\end{array}$ & $\begin{array}{c}26.58 \pm 4.54 \\
(18.40- \\
33.30)\end{array}$ & $\begin{array}{c}26.56 \pm 4.84 \\
(18.30-33.50)\end{array}$ & $\begin{array}{c}26.50 \pm 4.61 \\
(18.50-33.20)\end{array}$ \\
\hline $\begin{array}{c}\text { Transparency } \\
\text { (cm) }\end{array}$ & $\begin{array}{c}22.17 \pm 8.70 \\
(8.90- \\
39.80)\end{array}$ & $\begin{array}{l}29.34 \pm 12.00 \\
(8.10-48.70)\end{array}$ & $\begin{array}{l}22.18 \pm 13.00 \\
(9.10-44.60)\end{array}$ & $\begin{array}{c}21.39 \pm 9.33 \\
(9.10-38.00)\end{array}$ & $\begin{array}{c}23.94 \pm 7.39 \\
(9.40-36.90)\end{array}$ & $\begin{array}{c}18.94 \pm 5.81 \\
(8.20-31.50)\end{array}$ \\
\hline Depth (ft) & $\begin{array}{c}3.76 \pm 2.05 \\
(2.00-7.60)\end{array}$ & $\begin{array}{c}6.81 \pm 3.09 \\
(2.50-12.80)\end{array}$ & $\begin{array}{c}7.96 \pm 4.05 \\
(3.10-16.00)\end{array}$ & $\begin{array}{c}7.58 \pm 4.17 \\
(2.00-15.00)\end{array}$ & $\begin{array}{c}10.82 \pm 4.68 \\
(3.00-18.60)\end{array}$ & $\begin{array}{c}5.14 \pm 2.77 \\
(2.00-11.20)\end{array}$ \\
\hline $\begin{array}{c}\text { Dissolved oxygen } \\
(\mathrm{mg} / \mathrm{l})\end{array}$ & $\begin{array}{c}7.19 \pm 1.35 \\
(4.20-9.50)\end{array}$ & $\begin{array}{c}6.84 \pm 1.32 \\
(3.80-9.00)\end{array}$ & $\begin{array}{c}7.67 \pm 1.74 \\
(4.50-10.60)\end{array}$ & $\begin{array}{c}7.59 \pm 1.48 \\
(5.60-11.60)\end{array}$ & $\begin{array}{l}7.41 \pm 0.92 \\
(5.70-9.50)\end{array}$ & $\begin{array}{c}8.11 \pm 1.52 \\
(6.20-11.40)\end{array}$ \\
\hline pH & $\begin{array}{c}7.23 \pm 0.20 \\
(6.80-7.60)\end{array}$ & $\begin{array}{c}7.38 \pm 0.31 \\
(6.80-7.90)\end{array}$ & $\begin{array}{c}7.24 \pm 0.28 \\
(6.60-7.90)\end{array}$ & $\begin{array}{c}7.34 \pm 0.23 \\
(6.80-7.90)\end{array}$ & $\begin{array}{c}7.20 \pm 0.31 \\
(6.50-7.80)\end{array}$ & $\begin{array}{c}7.36 \pm 0.33 \\
(6.50-7.90)\end{array}$ \\
\hline Alkalinity (mg/l) & $\begin{array}{c}4.29 \pm 7.80 \\
(21.00- \\
57.00)\end{array}$ & $\begin{array}{c}42.00 \pm 10.03 \\
(17.00- \\
65.00)\end{array}$ & $\begin{array}{c}41.16 \pm 10.11 \\
(19.00- \\
57.00)\end{array}$ & $\begin{array}{c}40.38 \pm 8.29 \\
(23.00- \\
61.00)\end{array}$ & $\begin{array}{c}40.12 \pm 9.39 \\
(21.00-57.00)\end{array}$ & $\begin{array}{c}41.38 \pm 10.40 \\
(15.00-57.00)\end{array}$ \\
\hline $\mathrm{NO}_{3}-\mathrm{N}(\mathrm{mg} / \mathrm{l})$ & $\begin{array}{c}6.22 \pm 1.86 \\
(4.40-8.80)\end{array}$ & $\begin{array}{c}6.77 \pm 2.73 \\
(1.76-13.20)\end{array}$ & $\begin{array}{c}6.32 \pm 2.40 \\
(4.40-13.20)\end{array}$ & $\begin{array}{c}7.29 \pm 1.90 \\
(4.40-10.12)\end{array}$ & $\begin{array}{c}6.30 \pm 1.81 \\
(4.40-8.80)\end{array}$ & $\begin{array}{c}6.68 \pm 1.75 \\
(4.40-8.80)\end{array}$ \\
\hline $\mathrm{PO}_{4}-\mathrm{P}(\mathrm{mg} / \mathrm{l})$ & $\begin{array}{c}0.12 \pm 0.06 \\
(0.04-0.30)\end{array}$ & $\begin{array}{c}0.11 \pm 0.28 \\
(0.03-0.30)\end{array}$ & $\begin{array}{c}0.14 \pm 0.07 \\
(0.03-0.29)\end{array}$ & $\begin{array}{c}0.10 \pm 0.05 \\
(0.02-0.21)\end{array}$ & $\begin{array}{c}0.10 \pm 0.05 \\
(0.01-0.21)\end{array}$ & $\begin{array}{c}0.12 \pm 0.06 \\
(0.02-0.21)\end{array}$ \\
\hline
\end{tabular}

Table 4: Comparison of physical parameters (mean) recorded from six sampling sites by using ANOVA (N=48)

\begin{tabular}{|c|c|c|c|c|c|c|c|}
\hline \multirow[b]{2}{*}{ Parameters } & \multicolumn{6}{|c|}{ Sampling sites } & \multirow{2}{*}{$\begin{array}{c}\text { ANOVA } \\
\text { Significance }(P \\
\text { value })\end{array}$} \\
\hline & Site 1 & Site 2 & Site 3 & Site 4 & Site 5 & Site 6 & \\
\hline Air temperature ( $\mathrm{C}$ ) & $26.93^{\mathrm{b}}$ & $27.98^{\mathrm{ab}}$ & $29.62^{\mathrm{a}}$ & $29.20^{\mathrm{ab}}$ & $29.49^{\mathrm{ab}}$ & $30.33^{\mathrm{a}}$ & * \\
\hline $\begin{array}{c}\text { Water temperature } \\
\text { (C) }\end{array}$ & 25.60 & 25.53 & 26.66 & 26.58 & 26.56 & 26.50 & NS \\
\hline Transparency $(\mathbf{c m})$ & $22.17^{\mathrm{b}}$ & $29.34^{\mathrm{a}}$ & $22.18^{\mathrm{b}}$ & $21.39^{\mathrm{b}}$ & $23.94^{\mathrm{ab}}$ & $18.94^{\mathrm{b}}$ & * \\
\hline $\begin{array}{c}\text { Depth } \\
\text { (ft) }\end{array}$ & $3.76^{\mathrm{d}}$ & $6.81^{\mathrm{bc}}$ & $7.96^{\mathrm{b}}$ & $7.57^{\mathrm{b}}$ & $10.82^{\mathrm{a}}$ & $5.14^{\mathrm{cd}}$ & * \\
\hline
\end{tabular}

$\mathrm{NS}=$ Values are not significantly different $(P>0.05)$

* Values with different superscript letters in the same row indicate a significant difference at $5 \%$ significance level based on oneway ANOVA followed by Tukey's test. 


\section{Chemical Parameters}

\section{Dissolved oxygen}

From Table 3, dissolved oxygen (DO) concentration was found to vary from 4.20 to $9.50 \mathrm{mg} / \mathrm{l}, 3.80$ to $9.50 \mathrm{mg} / \mathrm{l}, 4.50$ to $10.60 \mathrm{mg} / \mathrm{l}, 5.60$ to $11.60 \mathrm{mg} / \mathrm{l}$, 5.70 to $9.50 \mathrm{mg} / \mathrm{l}$ and 6.20 to $11.40 \mathrm{mg} / \mathrm{l}$ during the experiment in sampling sites St.1, St.2, St.3, St.4, St.5, and St.6, respectively. The mean $( \pm \mathrm{SD})$ values of dissolved oxygen were $7.19 \pm 1.35 \mathrm{mg} / \mathrm{l}, 6.83 \pm$ $1.32 \mathrm{mg} / \mathrm{l}, 7.68 \pm 1.75 \mathrm{mg} / \mathrm{l}, 7.59 \pm 1.48 \mathrm{mg} / \mathrm{l}, 7.41 \pm$ $0.92 \mathrm{mg} / \mathrm{l}$ and $8.11 \pm 1.52 \mathrm{mg} / \mathrm{l}$ in sites St.1, St.2, St.3, St.4, St.5, and St.6, respectively (Table 3).

There was significant difference $(P<0.05)$ shown between St.1 and St.6 (Table 5).

\section{pH (Hydrogen ion concentration)}

$\mathrm{pH}$ values were found to fluctuate from 6.80 to 7.60 , 6.80 to $7.90,6.60$ to $7.90,6.80$ to $7.90,6.50$ to 7.80 and 6.50 to 7.90 in sampling sites St.1, St.2, St.3, St.4, St.5, and St.6, respectively (Table 3). The mean $( \pm \mathrm{SD})$ values of $\mathrm{pH}$ were $7.23 \pm 0.20,7.38 \pm 0.31$, $7.24 \pm 0.28,7.34 \pm 0.23,7.20 \pm 0.31$ and $7.36 \pm 0.33$ in sites St.1, St.2, St.3, St.4, St.5, and St.6, respectively (Table 3). There was significant difference $(P<0.05)$ was observed between St.5 and St.6 (Table 5).

\section{Total alkalinity}

In the present study the value of total alkalinity of the water in six sites of Dhepa River was found to fluctuate to a minimum $(15 \mathrm{mg} / \mathrm{l})$ in April in St.6 to a maximum $(65 \mathrm{mg} / \mathrm{l})$ in February in St.2 (Table 3). Total alkalinity of Dhepa River varied from 21 to 57 $\mathrm{mg} / \mathrm{l}, 17$ to $65 \mathrm{mg} / \mathrm{l}, 19$ to $57 \mathrm{mg} / \mathrm{l}, 23$ to $61 \mathrm{mg} / \mathrm{l}, 21$ to $57 \mathrm{mg} / \mathrm{l}$ and 15 to $57 \mathrm{mg} / \mathrm{l}$ during the experiment in sampling sites St.1, St.2, St.3, St.4, St.5, and St.6, respectively (Table 3$)$. From Table 3 mean $( \pm$ SD) values of total alkalinity were observed $43.29 \pm 7.80$ $\mathrm{mg} / \mathrm{l}, 42.00 \pm 10.03 \mathrm{mg} / \mathrm{l}, 41.16 \pm 10.12 \mathrm{mg} / \mathrm{l}, 40.37 \pm$ $8.29 \mathrm{mg} / \mathrm{l}, 40.13 \pm 9.39 \mathrm{mg} / \mathrm{l}$ and $41.38 \pm 10.41 \mathrm{mg} / \mathrm{l}$ in sites St.1, St.2, St.3, St.4, St.5, and St.6, respectively. There was no significant difference $(P>0.05)$ among different sites, when statistical analysis (ANOVA) was performed (Table 5).

Table 5: Comparison of chemical parameters (mean) recorded from six sampling sites by using ANOVA ( $=48)$

\begin{tabular}{|c|c|c|c|c|c|c|c|}
\hline \multirow{2}{*}{ Parameters } & \multicolumn{6}{|c|}{ Sampling sites } & \multirow{2}{*}{$\begin{array}{c}\text { ANOVA } \\
\text { Significance }(P \\
\text { value })\end{array}$} \\
\hline & Site 1 & Site 2 & Site 3 & Site 4 & Site 5 & Site 6 & \\
\hline $\begin{array}{c}\text { Dissolved } \\
\text { oxygen }(\mathrm{mg} / \mathrm{l})\end{array}$ & $7.19^{b}$ & $6.84^{\mathrm{ab}}$ & $7.67^{b}$ & $7.59^{\mathrm{ab}}$ & $7.41^{b}$ & $8.11^{\mathrm{a}}$ & $*$ \\
\hline pH & $7.23^{\mathrm{ab}}$ & $7.38^{\mathrm{a}}$ & $7.24^{\mathrm{ab}}$ & $7.34^{\mathrm{ab}}$ & $7.20^{\mathrm{b}}$ & $7.36^{\mathrm{a}}$ & $*$ \\
\hline Alkalinity (mg/l) & 4.29 & 42.00 & 41.16 & 40.38 & 40.12 & 41.38 & NS \\
\hline $\mathrm{NO}_{3}-\mathrm{N}(\mathrm{mg} / \mathrm{l})$ & 6.22 & 6.77 & 6.32 & 7.29 & 6.30 & 6.68 & NS \\
\hline $\mathrm{PO}_{4}-\mathrm{P}(\mathrm{mg} / \mathrm{l})$ & $0.12^{\mathrm{ab}}$ & $0.11^{\mathrm{ab}}$ & $0.14^{\mathrm{a}}$ & $0.10^{\mathrm{b}}$ & $0.10^{\mathrm{b}}$ & $0.12^{\mathrm{ab}}$ & * \\
\hline
\end{tabular}

NS $=$ Values are not significantly different $(P>0.05)$

* Values with different superscript letters in the same row indicate a significant difference at $5 \%$ significance level based on oneway ANOVA followed by Tukey's test.

\section{$\mathrm{NO}_{3}-\mathrm{N}$ (Nitrate-nitrogen)}

The concentrations of nitrate-nitrogen in all sampling sites were more or less similar. The ranges of nitratenitrogen were from 4.40 to $8.80 \mathrm{mg} / \mathrm{l}, 1.76$ to 13.20 $\mathrm{mg} / \mathrm{l}, 4.40$ to $13.20 \mathrm{mg} / \mathrm{l}, 4.40$ to $10.12 \mathrm{mg} / \mathrm{l}, 4.40$ to
$8.80 \mathrm{mg} / \mathrm{l}$ and 4.40 to $8.80 \mathrm{mg} / \mathrm{l}$ in sampling sites St.1, St.2, St.3, St.4, St.5, and St.6, respectively (Table 3). The highest $(13.20 \mathrm{mg} / \mathrm{l})$ and lowest $(1.76 \mathrm{mg} / \mathrm{l})$ value of nitrate-nitrogen was observed in St.2 in February and March, respectively and highest value (13.20 
$\mathrm{mg} / \mathrm{l}$ ) was observed in St.3 also in the month of February. The mean $( \pm \mathrm{SD})$ values of nitrate-nitrogen were $6.23 \pm 1.86 \mathrm{mg} / \mathrm{l}, 6.77 \pm 2.74 \mathrm{mg} / \mathrm{l}, 7.33 \pm 2.40$ $\mathrm{mg} / \mathrm{l}, 7.29 \pm 1.90 \mathrm{mg} / \mathrm{l}, 6.80 \pm 1.81 \mathrm{mg} / \mathrm{l}$ and $6.68 \pm$ $1.75 \mathrm{mg} / \mathrm{l}$ in sites St.1, St.2, St.3, St.4, St.5, and St.6, respectively (Table 3 ). There was no significant difference $(P>0.05)$ was observed among different sites (Table 5).

\section{$\mathrm{PO}_{4}-\mathrm{P}$ (Phosphate-phosphorous)}

In the present study the highest $\mathrm{PO}_{4}-\mathrm{P}(0.30 \mathrm{mg} / \mathrm{l})$ concentration was found in November in St.1 and St.2 and the lowest phosphate-phosphorous concentration $(0.01 \mathrm{mg} / \mathrm{l})$ was found in April in St.5. The ranges of phosphate-phosphorous were from 0.04 to $0.30 \mathrm{mg} / \mathrm{l}$, 0.03 to $0.30 \mathrm{mg} / \mathrm{l}, 0.03$ to $0.29 \mathrm{mg} / \mathrm{l}, 0.02$ to 0.21 $\mathrm{mg} / \mathrm{l}, 0.01$ to $0.21 \mathrm{mg} / \mathrm{l}$ and 0.02 to $0.21 \mathrm{mg} / \mathrm{l}$ in sampling sites St.1, St.2, St.3, St.4, St.5, and St.6, respectively (Table 3$)$. The mean $( \pm \mathrm{SD})$ values of phosphate-phosphorous were $0.12 \pm 0.05 \mathrm{mg} / \mathrm{l}, 0.11 \pm$ $0.28 \mathrm{mg} / \mathrm{l}, 0.14 \pm 0.07 \mathrm{mg} / \mathrm{l}, 0.10 \pm 0.05 \mathrm{mg} / \mathrm{l}, 0.11 \pm$ $0.05 \mathrm{mg} / \mathrm{l}$ and $0.12 \pm 0.07 \mathrm{mg} / \mathrm{l}$ in sites St.1, St.2, St.3, St.4, St.5, and St.6, respectively (Table 3). St.3, St.4 and St.5 varied significantly (Table 5). However, there was no significant difference was observed in St.1, St.2 and St.6. Average fortnightly variations of phosphate-phosphorous among different sites.

\section{Discussion}

The suitable water quality parameters are prerequisite for healthy aquatic environment and for the production of sufficient fish food organisms. Primary productivity of a waterbody depends on the physical, chemical and others factors of the environment (Rahman, 1992).

Water temperature is one of the most important among the external factors which influence the aquatic ecology (Huet, 1986). During the current study temperature varied between 17.00 to $33.50{ }^{\circ} \mathrm{C}$ of which maximum value $\left(33.50^{\circ} \mathrm{C}\right)$ was noticed in April in St.5 and the minimum value $\left(17.00{ }^{\circ} \mathrm{C}\right)$ in December in St. 2 mainly in line with changes in weather conditions. Statistical analysis revealed no significance differences $(P>0.05)$ in temperatures among all sampling sites. In addition, the variations in water temperature may also be attributed to water level fluctuations due to use of water for irrigation and for dry season. The variations were further aggravated by heavy precipitation during the monsoon. The range of water temperature of Dhepa river is not similar to that obtained for river Talar 10.10- $29.7^{\circ} \mathrm{C}$ (Alavi and Jafari, 2010) and for river Mouri 22.1- $23.5^{\circ} \mathrm{C}$ (Khan et al., 2007). These might be different geological position of this river.
Transparency is another important environmental factor. In the present study transparency was found to vary from 8.10 to $48.70 \mathrm{~cm}$. The highest value $(48.70$ $\mathrm{cm}$ ) was reported in February in St.2 whiles the lower value $(8.10 \mathrm{~cm})$ was also reported in $\mathrm{St} .2$ in September. Transparency was not varied significantly according to the sites except St.2 (Table 4) may be due to fluctuation of water level. Throughout the period of study the average value of Dhepa river transparency was $22.99 \mathrm{~cm}$ while in Shibsha and Buriganga River the average value were $37.25 \mathrm{~cm}$ (Khan et al., 2007 and Hossain, 1992).

Depth is another important factor in limnological studies. Highest depth in Dhepa River was observed in September in St.5 and lowest water level was observed in April in St.6. Water level fluctuation in Dhepa River mainly in the line of seasonal changes and geological position. Concentration of nutrients will increase in lower level of water especially in winter season. This was also happened in case of Dhepa River. Singh et al. (2010) observed highest water depth in monsoon and lowest in summer season in Manipur river. This finding is similar to the finding of the present study.

Dissolved oxygen is another important parameter of water quality, directly affecting survival and distributing flora and fauna in an ecosystem. In the present study dissolved oxygen values ranged from 3.80 to $11.60 \mathrm{mg} / \mathrm{l}$ of which greatest amount $(11.60 \mathrm{mg} / \mathrm{l})$ was noted in December in St.4 and lowest amount $(3.80 \mathrm{mg} / \mathrm{l})$ in April in St.2. The quantity of DO in water is directly or indirectly dependent on water temperature. Minimum dissolved oxygen concentration has to be at least $5.00 \mathrm{mg} / \mathrm{l}$ for maintaining aquatic life in healthy condition and dissolved oxygen concentration less than $5.00 \mathrm{mg} / \mathrm{l}$ are indicative of pollution (Khandaker, 1986). During the study period in St.2 dissolved oxygen concentration was low $(3.80 \mathrm{mg} / \mathrm{l})$ which might be higher level of pollution arise from organic pollution by washing of mechanized vessels, urban area nearer to the river bank, anthropogenic disturbances and use of fertilizer etc or may be due to weather condition. There was significant difference observed between St.2 and St.6 (Table 5). Dissolved oxygen concentration in St.1, St.3, St.4, St.5 and St.6 was above $5 \mathrm{mg} / \mathrm{l}$ which indicate a better condition for aquatic organisms. In the Buriganga River DO concentration was found $2-3 \mathrm{mg} / \mathrm{l}$ that indicates high rate of pollution (Moniruzzaman et al., 2009). Khandaker (1986) recorded $5.1 \mathrm{mg} / \mathrm{l}$ dissolved oxygen in Karnafully river which is lower $(7.47 \mathrm{mg} / \mathrm{l})$ from the findings of the present study. 
$\mathrm{pH}$ is considered as one of the most important chemical parameter of water since most of the aquatic organisms are adapted to an average $\mathrm{pH}$. The $\mathrm{pH}$ values during the study ranged from 6.50 to 7.90. Slightly alkaline water (7.90) observed in all sites in March, this may be due to low level of water and slightly acidic value (6.50) also observed in all sites in September. The $\mathrm{pH}$ value in the present investigation remained a buffer condition. Begum and Khanam (2009) observed 6.6-8.0 pH in Shitalakhya river water which is similar to the present study. The $\mathrm{pH}$ value (8.3-8.4) in Hoogly river by Roy (1955) and (7.3-8.3) in Mouri river by Khan et al. (2007) also showed a buffer condition which are in agreement with the finding of the present study.

Total alkalinity values in the current study ranged between 15.00 to $65.00 \mathrm{mg} / \mathrm{l}$. Maximum and minimum value of alkalinity found in the month of February in St. 2 and April in St.6, respectively. There was no significant difference found among the sampling sites. Maximum and minimum value in total alkalinity might be accounted for the depletion in water level and showed a definite trend in seasonal fluctuation among the six sampling sites. Similar seasonal fluctuation in total alkalinity in Talar River, Iran was also observed by Alavi and Jafari (2010).

Phosphate is an important nutrient for the maintenance of fertility of water body. During the present investigation phosphate-phosphorous concentration ranged from 0.01 to $0.30 \mathrm{mg} / \mathrm{l}$. The average $\mathrm{PO}_{4}-\mathrm{P}$ concentration in all sampling sites was comparatively similar. The highest concentration $(0.30 \mathrm{mg} / \mathrm{l})$ was observed in November in St.1 and St. 2 and lowest concentration $(0.01 \mathrm{mg} / \mathrm{l})$ in April in St.5. These higher and lower values may be due to depletion in water level and for seasonal fluctuation. Shah et al. (2008) was found 0.9 to $1.9 \mathrm{mg} / 1 \mathrm{PO}_{4}-\mathrm{P}$ concentration in Shibsha River which is higher than the findings of the present study. Phosphatephosphorous concentration was found to vary from 5 to $10 \mathrm{mg} / \mathrm{l}$ in Mouri River which indicate medium to high rate of pollution (Khan et al., 2007). However, $\mathrm{PO}_{4}-\mathrm{P}$ concentration in Dhepa River was less than one. So the productivity status of Dhepa River is in good condition.

Nitrogen is a limiting factor and very important macronutrient for microalgae in aquaculture and fish production. Among various forms of nitrogenous nutrients, nitrate is most important. It is the available form of nitrogen to phytoplankton and other plants. Typical levels in surface waters range from 0.005 to $0.5 \mathrm{mg} / \mathrm{l}$ nitrate. However, nitrate is relatively nontoxic to fish and is not a health hazard except at exceedingly high levels (above $90 \mathrm{mg} / \mathrm{NO}_{3}-\mathrm{N}$ ) (Nathan and Hugh, 1977). During flood times and organic pollution, nitrate-nitrogen content may be expected to increase significantly. In the present study $\mathrm{NO}_{3}-\mathrm{N}$ concentration ranged from 1.76 to $13.20 \mathrm{mg} / \mathrm{l}$. Urban area and use of fertilizers in agricultural land on the bank of the river in St.1 and St.2, washing of community center clothes with detergents in St.3, humane waste, through of dead animal in these three sites may be the reason behind the higher $\mathrm{NO}_{3}-\mathrm{N}$ concentration. Use of fertilizers and domestic and human waste in St.4, 5 and 6 may be also responsible for higher $\mathrm{NO}_{3}-\mathrm{N}$ concentration. Seasonal fluctuation and lower level of water may also be the reason for higher concentration.

The highest concentrations of $\mathrm{NO}_{3}-\mathrm{N}$ were generally found in the large rivers. Rankin et al. (1999) stated: "While nutrient concentrations are expected to increase in the larger mainstream rivers, the concentrations are themselves indicative of enrichment that is largely the product of anthropogenic sources and activities." Higher level of $\mathrm{NO}_{3}-\mathrm{N}(1.85 \mathrm{mg} / \mathrm{l})$ for pollution was observed by Arimoro et al. (2008) in tropical stream. The range of $\mathrm{NO}_{3}-\mathrm{N}$ concentration was 0.3 to $0.7 \mathrm{mg} / \mathrm{l}$ in Shibsha River recorded by Shah et al. (2008). However, result of the present findings is not similar to the above investigation.

\section{Conclusion}

Among the value of different parameters water temperature $\left(17-33.50{ }^{\circ} \mathrm{C}\right)$ and alkalinity (15-65 $\mathrm{mg} / \mathrm{l})$ in six sampling sites were not varied significantly. Transparency range was $(8.10-48.7 \mathrm{~cm})$, highest and lowest value found in St.2 in February and September respectively. Highest water depth was observed in the month of September and lowest in April. The $\mathrm{pH}$ value of Dhepa river was slightly acidic to slightly alkaline in all sites. Highest dissolved oxygen concentration (11.60 mg/l) was observed in December and lowest concentration (3.80 $\mathrm{mg} / \mathrm{l})$ was found in April. Dissolved oxygen concentrations in all sites except St.2 in March are suitable for fish culture. Nitrate-nitrogen concentrations in all sites are more than $10 \mathrm{mg} / \mathrm{l}$ because of anthropogenic activities. In low water temperature phosphate-phosphorous concentration was high. 


\section{References}

Alavi, S. S. and Jafari. N. 2010. Phytoplankton community in relation to physico-chemical characteristics of the Talar River, Iran. Journal of Applied Science Environment and Managemen, 14(2): 51 - 56.

APHA. 1992. Standard Methods for the Examination of Water and Waste Water. $18^{\text {th }}$ ed. American Public Health Association, Washington DC. p. 1268.

Arimoro, F. O.; Edema N. E. and Amaka. R. O. 2008. Phytoplankton community responses in a perturbed tropical stream in the Niger delta, Nigeria. Tropical Freshwater Biology., 17(1): 37-52.

Begum, Z. N. T.; and Khanam, D. 2009. Physicochemical aspects and phytoplankton of the river Shitalakhya receiving pharmaceutical effluents. Bangladesh Journal of Botany, 38(1): 77-85.

De Pauw, N. and Vanhooren. G. 1983. Method for biological quality assessment of water courses in Belgium. Hydrobiologia, 100: 153-168.

Hossain, M. T. 1992. Study on the environmental impact assessment of the effluent discharge by the Chittagong urea fertilizer limited, in the Karnafully river estuary. M.S. thesis, Institute of Marine Science, University of Chittagong, Bangladsh. p. 56.

Huet, M. 1986. Text book of fish culture: breeding and cultivation of fish. Fishing News Books, Farnham, United Kingdom. p. 438.

Khan, A. N.; Kamal, D. Rahman, M. A. Ahmed, F. 2007. Stydy on the physico-chemical properties of water of Mouri river, Khulna, Bangladesh. Pakistan Journal of Biological Sciences, 10(5): 710-717.

Khandakar, A. T. 1986. Industrial and marine pollution in coastal areas of Bangladesh. Part
Report of Case Study in Bangladesh, Conducted by ESCAP.

Manjare, S. A.; Vhanalakar, S. A. and Muley, D.V. 2010. Analysis of water quality using physico-chemical parameters Tamdalge Tank in Kolhapur district, Maharashtra, India. International Journal of Advanced Biotechnology and Research, 1(2):115-119.

Moniruzzaman, M.; Elahi, S. F. and Jahangir, M. A. A. 2009. Study on temporal variation of physico-chemical parameters of Buriganga river water through GIS (Geographical Information System) technology. Bangladesh Journal of Scientific and Industrial Research, 44(3): 327-334.

Nathan, M. S. and Hugh. K. T. 1977. Understanding of your fish pond water analysis report, FSA9090. University of Arkansas at Pine Bluff, Pine Bluff, Arkansas.

Rahman, M.S. 1992. Water quality management in aquaculture, BRAC prokashana, Dhaka. pp. 83-84.

Rankin, E.; Miltner, B. Yoder, C. and Mishne, D. 1999. Association between nutrients, habitat and the aquatic biota in Ohio rivers and streams, Ohio. p. 70.

Roy, H. K. 1955. Plankton ecology of river Hoogly in Patna, West Bengal. Ecology. 36: 169-175.

Shah, M. M. R.; Hossain, M. Y.; Begum, M.; Ahmed, Z. F.; Ohtomi, J.; Rahman, M. M.; Alam, M. J.; Islam M. A. and Fulanda B. 2008. Seasonal variations of phytoplanktonic community structure and production in relation to environmental factors of the southwest coastal waters of Bangladesh. Journal of Fisheries and Aquatic Science, 3(2): 102-113.

Singh M. R.; Gupta, Asha and Beeteswari, K. 2010. Physico-chemical properties of water samples from Manipur river system, India. Journal of Applied Science Environment and Management, 14(4): 85-89. 